PROCEEDINGS OF THE

AMERICAN MATHEMATICAL SOCIETY

Volume 134, Number 1, Pages 67-74

S 0002-9939(05)07948-7

Article electronically published on August 12, 2005

\title{
WEAKLY NULL SEQUENCES WITH AN UNCONDITIONAL SUBSEQUENCE
}

\author{
ALEXANDER D. ARVANITAKIS
}

(Communicated by N. Tomczak-Jaegermann)

\begin{abstract}
In the present paper we provide sufficient conditions such that a normalized pointwise convergent to zero sequence in $C(K, X)$ with $K$ a compact space and $X$ a Banach space has an unconditional subsequence.

As a consequence we obtain that any such sequence of functions $\left(f_{n}\right)_{n}$ with finite and uniformly bounded cardinality of their range admits an unconditional subsequence.
\end{abstract}

\section{INTRODUCTION}

The following theorem is due to H. Rosenthal [9], and its initial proof uses transfinite induction.

Theorem 1.1 (H. Rosenthal). Let $\left(f_{n}\right)_{n \in \mathbb{N}}$ be a sequence of non-zero characteristic functions in $C(K)$ with $K$ a compact Hausdorff space. If $\left(f_{n}\right)_{n}$ converges pointwise to zero, then it contains an unconditional basic subsequence.

Maurey-Rosenthal's example 8 shows that there is a compact $K$ and a pointwise null sequence $f_{n} \in C(K),\left(f_{n}\right)_{n}$ has no unconditional basic subsequence. The range of every $f_{n}$ is a countable set $\{0\} \cup\left\{r_{k}: k \in \mathbb{N}\right\}$ where $\left\{r_{k}\right\}_{k}$ is a strictly decreasing null sequence. Setting in this case

$$
g_{n}(x)= \begin{cases}f_{n}(x), & \text { if } f_{n}(x)=r_{1}, \ldots, r_{n}, \\ 0, & \text { otherwise }\end{cases}
$$

we get that $\left\|g_{n}-f_{n}\right\| \leq r_{n+1} \rightarrow 0$. Therefore also $\left(g_{n}\right)_{n}$ has no unconditional basic subsequence yielding that Theorem 1.1 cannot be extended in this case. So it is natural to ask whether it can be extended in the case where the cardinality of the range of $f_{n}$ is finite and uniformly bounded by some positive integer.

In this direction, we prove the following:

Theorem 1.2. Let $K$ be a Hausdorff compact space, let $X$ be a Banach space and let $f_{n}: K \rightarrow X, n \in \mathbb{N}$, be a sequence of normalized pointwise null continuous functions with the property that their range is of finite cardinality uniformly bounded by some positive integer $J$. Then $\left(f_{n}\right)_{n}$ has an unconditional subsequence.

Received by the editors April 2, 2004 and, in revised form, September 1, 2004.

2000 Mathematics Subject Classification. Primary 05D10, 46B15.

The author was partially supported by EPEAEK research program Pythagoras. 
In the case where $X$ is finite dimensional, we obtain the following stronger result.

Theorem 1.3. Let $K$ be a compact space and let $f_{m}: K \rightarrow \mathbb{R}^{n}, m \in \mathbb{N}$, be a uniformly bounded sequence of continuous functions on $K$ which is pointwise convergent to zero. Assume moreover that there exists a sequence of positive numbers $\epsilon_{m}$ converging to zero and a positive number $\mu$ such that for every $m \in \mathbb{N}$ and $x \in K$ either $\left\|f_{m}(x)\right\| \leq \epsilon_{m}$ or $\left\|f_{m}(x)\right\| \geq \mu$. Then $\left(f_{m}\right)_{m}$ has an unconditional basic subsequence.

To derive these we make use of a combinatorial theorem also proved in this paper which states the following:

Theorem 1.4. Assume that $I$ is a set, $n$ a positive integer and for any $i \in I$, $G_{1}^{i}, \ldots, G_{n}^{i}$ finite subsets of $\mathbb{N}$, such that setting $G^{i}=\bigcup_{k=1}^{n} G_{k}^{i}$, the closure of the family $\left\{G^{i}: i \in I\right\}$ in the pointwise topology consists of finite subsets of $\mathbb{N}$. Then for any $L \in[\mathbb{N}]$, there is $N=\left\{n_{1}<n_{2}<\ldots\right\} \in[L]$ such that the following holds:

Given $i \in I, q \in \mathbb{N}, k \in\{1, \ldots, n\}$ and $A \subset G_{k}^{i} \cap\left\{n_{1}, \ldots, n_{q}\right\}$, there is $i^{\prime} \in I$ such that $G_{k}^{i^{\prime}} \cap\left\{n_{1}, \ldots, n_{p}\right\}=A$ and for all $k^{\prime} \neq k, G_{k^{\prime}}^{i^{\prime}} \cap\left\{n_{1}, \ldots, n_{q}\right\} \subset A$.

Here by $[L]$ we denote the set of all infinite subsets of $L$.

The above theorem makes use of some ideas due to J. Elton [4] and it shares similar arguments with the proofs of two results concerning restricted forms of unconditionality that occur in weakly null sequences. Namely J. Elton's near unconditionality (4]) and Argyros-Mercourakis-Tsarpalias convex unconditionality [2]. It extends an earlier result in [2] which was used for an alternative proof of the above-mentioned Rosenthal's Theorem. This proof also can be found in [1].

After submitting the present paper, two recent papers came to our attention: The first one is due to I. Gasparis, E. Odell and B. Wahl, 6], where it is proved among others, the case $n=1$ of Theorem 1.3. Some additional information is also obtained about the norm of the space generated by the subsequence. The second, due to J. Lopez-Abad and S. Todorcevic [7] (see also [11]) also provides a proof for the case $n=1$ of Theorem 1.3 and also a proof for Theorem 1.2 in the case $X=\mathbb{R}$. Both papers have a different approach to these problems than ours.

\section{Some COMbinatorial RESUlts}

We make use of the following important principle of infinite combinatorics, namely the infinite Ramsey theorem. For $M$ an infinite subset of $\mathbb{N}$, we denote by $[M]$ the set of all infinite subsets of $M$. We endow $[\mathbb{N}]$ with the topology of pointwise convergence.

Theorem 2.1 ([3, 10, 5]). Let $\mathcal{A}$ be an analytic subset of $[\mathbb{N}]$. For every $L \in[\mathbb{N}]$ there exists an $M \in[L]$ such that either $[M] \subset \mathcal{A}$ or else $[M] \subset[L] \backslash \mathcal{A}$.

We pass now to the proof of Theorem 1.4

Proof of Theorem 1.4. For $1 \leq k \leq n$ let $P_{k}$ be the following property of $L_{k}=$ $\left\{\ell_{1}<\ell_{2}<\ldots\right\}$.

For all $i \in I$ and $q \in \mathbb{N}$ if $A \subset G_{k}^{i} \cap\left\{\ell_{1}, \ldots, \ell_{q}\right\}$, then there is $i^{\prime} \in I$ such that $G_{k}^{i^{\prime}} \cap\left\{\ell_{1}, \ldots, \ell_{q}\right\}=A$ and for all $k^{\prime} \neq k, G_{k^{\prime}}^{i^{\prime}} \cap\left\{\ell_{1}, \ldots, \ell_{q}\right\} \subset A$.

We first prove that for a fixed $k \in\{1, \ldots, n\}$ and $L \in[\mathbb{N}]$ there is $N \in[L]$ satisfying property $P_{k}$. So, we fix $k$ and prove the following claim. 
Claim. For any $L \in[\mathbb{N}]$ and $J \subset I$ we partition $[L]$ into two disjoint sets $\mathcal{A}_{J, L}$ and $\mathcal{B}_{J, L}$ as follows:

$M=\left\{m_{1}<m_{2}<\ldots\right\} \in \mathcal{A}_{J, L}$ if and only if for all $q \in \mathbb{N}$, if there exists an $i \in J$ such that $\left\{m_{2}, \ldots, m_{q}\right\} \subset G_{k}^{i}$, then there exists an $i^{\prime} \in J$ such that $\left\{m_{2}, \ldots, m_{q}\right\} \subset G_{k}^{i^{\prime}}$ and moreover $m_{1} \notin \bigcup_{k^{\prime}=1}^{n} G_{k^{\prime}}^{i^{\prime}}$.

We also set $\mathcal{B}_{J, L}=[L] \backslash \mathcal{A}$.

We claim that there is an $M \in[L]$ such that $[M] \subset \mathcal{A}_{J, L}$.

It is easy to check that if $M=\left\{m_{1}<m_{2}<\ldots\right\} \in \mathcal{B}_{J, L}$, then there is a $q \in \mathbb{N}$ such that any other set starting with $m_{1}, m_{2}, \ldots, m_{q}$ is also in $\mathcal{B}_{J, L}$. Therefore $\mathcal{B}_{J, L}$ is open, so we can find an $M=\left\{m_{1}<m_{2}<\ldots\right\} \in[L]$ such that either $[M] \subset \mathcal{A}_{J, L}$ or $[M] \subset \mathcal{B}_{J, L}$. Next we argue that the second case is impossible, by showing that for any $p \in \mathbb{N}$, there is an $i \in I$ such that $\left\{m_{1}, \ldots, m_{p}\right\} \subset \bigcup_{k^{\prime}=1}^{n} G_{k^{\prime}}^{i}$. This contradicts the fact that $\left\{G^{i}=\bigcup_{k^{\prime}=1}^{n} G_{k^{\prime}}^{i}: i \in I\right\}$ is relatively compact in the finite subsets of $\mathbb{N}$. So fix such a $p$ and note that for all $\ell \in[1, p]$, the set $\left\{m_{\ell}, m_{p+1}, m_{p+2}, \ldots\right\}$ is in $[M]$. Therefore, there exists a $q(\ell) \geq p$ and an $i(\ell) \in J$ so that $\left\{m_{p+1}, \ldots, m_{q(\ell)}\right\} \subset$ $G_{k}^{i(\ell)}$ and whenever for some $i^{\prime} \in J,\left\{m_{p+1}, \ldots, m_{q(\ell)}\right\} \subset G_{k}^{i^{\prime}}$, we have that also $m_{\ell} \in \bigcup_{k^{\prime}=1}^{n} G_{k^{\prime}}^{i^{\prime}}$. Let $q\left(\ell_{r}\right)$ be the greatest among $q(1), \ldots, q(p)$. Then for all $\ell$, $\left\{m_{p+1}, \ldots, m_{q(\ell)}\right\} \subset G_{k}^{i\left(\ell_{r}\right)}$ and therefore $\left\{m_{1}, \ldots, m_{p}\right\} \subset \bigcup_{k^{\prime}=1}^{n} G_{k^{\prime}}^{i\left(\ell_{r}\right)}$.

It follows therefore that for some $M=\left\{m_{1}<m_{2}<\ldots\right\} \in[L],[M] \subset \mathcal{A}_{J, L}$.

Using the previous claim, we define inductively $M_{1} \supset M_{2} \supset \ldots$ infinite subsets of $L$ and $n_{1}=\min M_{1}<n_{2}=\min M_{2}<\ldots$, as follows:

$M_{1}$ is the appropriate set given by the claim such that $\left[M_{1}\right] \subset \mathcal{A}_{I, L}$.

Assume we have defined $M_{1} \supset M_{2} \supset \cdots \supset M_{p}$ and $n_{1}=\min M_{1}<n_{2}=$ $\min M_{2}<\cdots<n_{p}=\min M_{p}$. Let $F_{1}, F_{2}, \ldots, F_{2^{p}}$ be an enumeration of the subsets of $\left\{n_{1}, \ldots, n_{p}\right\}$ and for $1 \leq j \leq 2^{p}$, let

$I_{F_{j}}=\left\{i \in I: G_{k}^{i} \cap\left\{n_{1}, \ldots, n_{p}\right\}=F_{j}\right.$ and for all $\left.k^{\prime} \neq k, G_{k^{\prime}}^{i} \cap\left\{n_{1}, \ldots, n_{p}\right\} \subset F_{j}\right\}$.

Repeatedly using the previous claim, let $M_{p}^{1} \in\left[M_{p} \backslash\left\{n_{p}\right\}\right]$ be such that $\left[M_{p}^{1}\right] \subset$ $\mathcal{A}_{I_{F_{1}}, M_{p} \backslash\left\{n_{p}\right\}}, M_{p}^{2} \in\left[M_{p}^{1}\right]$ be such that $\left[M_{p}^{2}\right] \subset \mathcal{A}_{I_{F_{2}}, M_{p}^{1}}, \ldots, M_{p}^{2^{p}}$ be such that $\left[M_{p}^{2^{p}}\right] \subset \mathcal{A}_{I_{F^{2}}, M_{p}^{2 p}-1}$. We set $M_{p+1}=M_{p}^{2^{p}}$ and $n_{p+1}=\min M_{p+1}>n_{p}$. Observe that since $M_{p}^{1} \supset M_{p}^{2} \supset \ldots, \supset M_{p}^{2^{p}}=M_{p+1}$, we have that

$$
\left[M_{p+1}\right] \subset \mathcal{A}_{I_{F_{j}}, M_{p}} \text { for all } 1 \leq j \leq 2^{p} .
$$

To prove that $N=\left\{n_{1}<n_{2}<\ldots\right\}$ has property $P_{k}$, let $i \in I, q \in \mathbb{N}$ and $A \subset G_{k}^{i} \cap\left\{n_{1}, \ldots, n_{q}\right\}$. We find inductively on $p \leq q, i_{p} \in I$ such that

$$
\begin{aligned}
G_{k}^{i_{p}} \cap\left\{n_{1}, \ldots, n_{p}\right\} & =A \cap\left\{n_{1}, \ldots, n_{p}\right\}, \\
\text { for } k^{\prime} \neq k, G_{k^{\prime}}^{i_{p}} \cap\left\{n_{1}, \ldots, n_{p}\right\} & \subset A \cap\left\{n_{1}, \ldots, n_{p}\right\} \\
\text { and } G_{k}^{i_{p}} \cap\left\{n_{p+1}, \ldots, n_{q}\right\} & \supset A \cap\left\{n_{p+1}, \ldots, n_{q}\right\} .
\end{aligned}
$$

Then $i_{q}$ is the required index in $I$, demonstrating that $N$ indeed has property $P_{k}$.

To start with, set $i_{0}=i$. Then (4) is the only non-void condition and is satisfied by our hypothesis that $A \subset G_{k}^{i} \cap\left\{n_{1}, \ldots, n_{q}\right\}$. Assume we have defined $i_{p}$ so that 
(2), (3) and (4) are fulfilled, and $p<q$. Consider two cases:

If $n_{p+1} \in A$, then by (4) $n_{p+1} \in G_{k}^{i_{p}}$, thus setting $i_{p+1}=i_{p}$, (2), (3) and (4) are also fulfilled for $p+1$.

As a second case, let $n_{p+1} \notin A$. Set $F_{1}=G_{k}^{i_{p}} \cap\left\{n_{1}, \ldots, n_{p}\right\}$ and let

$Q=\left\{n_{p+1}\right\} \cup\left\{n \in N: n \in A \cap\left\{n_{p+1}, \ldots, n_{q}\right\}\right.$ or $\left.n>\max A \cap\left\{n_{p+1}, \ldots, n_{q}\right\}\right\}$

be the infinite subset of $N$ beginning with $\left\{n_{p+1}\right\} \cup\left(A \cap\left\{n_{p+1}, \ldots, n_{q}\right\}\right)$. Since by (1) $\left[M_{p+1}\right] \subset \mathcal{A}_{I_{F_{1}}, M_{p}}$ and $Q \in\left[M_{p+1}\right]$, we get that there is an $i_{p+1} \in I_{F_{1}}$ such that $G_{k}^{i_{p+1}} \supset A \cap\left\{n_{p+1}, \ldots, n_{q}\right\}=A \cap\left\{n_{p+2}, \ldots, n_{q}\right\}$ (which demonstrates (44) for $p+1)$, but now $n_{p+1} \notin \bigcup_{k^{\prime}=1}^{n} G_{k^{\prime}}^{i_{p+1}}$. Since moreover $i_{p+1} \in I_{F_{1}}$ and $F_{1}=$ $G_{k}^{i_{p}} \cap\left\{n_{1}, \ldots, n_{p}\right\}$, we get that $G_{k}^{i_{p+1}} \cap\left\{n_{1}, \ldots, n_{p}\right\}=A \cap\left\{n_{1}, \ldots, n_{p}\right\}$ and for $k^{\prime} \neq k, G_{k^{\prime}}^{i_{p+1}} \cap\left\{n_{1}, \ldots, n_{p}\right\} \subset A \cap\left\{n_{1}, \ldots, n_{p}\right\}$. This immediately implies (2) and (3) for $p+1$, since $n_{p+1}$ does not belong to any of the sets $G_{1}^{i_{p+1}}, \ldots, G_{n}^{i_{p+1}}$ or $A$.

So, for a fixed $k \in\{1, \ldots, n\}$ and $L \in[\mathbb{N}]$ there is $L_{k} \in[L]$ satisfying property $P_{k}$. Note that in this case, if $L^{\prime} \in\left[L_{k}\right]$, then $L^{\prime}$ also satisfies property $P_{k}$. This is true since if $L^{\prime}=\left\{\ell_{k_{1}}, \ell_{k_{2}}, \ldots\right\}$ with $k_{1}<k_{2}<\ldots$, and $A \subset G_{k}^{i} \cap\left\{\ell_{k_{1}}, \ell_{k_{2}}, \ldots, \ell_{k_{q}}\right\}$, for some $i$ and $q$, then also $A \subset G_{k}^{i} \cap\left\{\ell_{1}, \ldots, \ell_{k_{q}}\right\}$ and we may apply property $P_{k}$ for $L_{k}$ to find the appropriate index $i^{\prime}$. Using this observation we successively find $L_{1} \in[L], L_{2} \in\left[L_{1}\right], \ldots, L_{n} \in\left[L_{n-1}\right]$ such that $L_{k}$ satisfies property $P_{k}$. Then $M=L_{n}$ satisfies property $P_{k}$ for any $k$ which is exactly what we wanted to prove.

Using stronger hypothesis about the compactness of $G_{1}^{i}, \ldots, G_{n}^{i}, i \in I$, we obtain the following result:

Corollary 2.2. Assume that $I$ is a set, $n$ a positive integer and $\left\{\left(G_{k}^{i}\right)_{k=1}^{n}: i \in I\right\}$ a compact family of $\left(\{0,1\}^{\mathbb{N}}\right)^{n}$. Then for any $L \in[\mathbb{N}]$, there is $N \in[L]$ such that the following holds:

Given $i \in I, k=1, \ldots, n$ and $A \subset G_{k}^{i} \cap N$, then there is $i^{\prime} \in I$ such that $G_{k}^{i^{\prime}} \cap N=A$ and $G_{k^{\prime}}^{i^{\prime}} \cap N \subset A$ whenever $k^{\prime} \neq k$.

In particular, if for every $i, G_{1}^{i}, G_{2}^{i}, \ldots, G_{n}^{i}$ are pairwise disjoint and for some $i$ and $k, A \subset G_{k}^{i}$, then there is $i^{\prime} \in I$ such that $G_{k}^{i^{\prime}} \cap N=A$ and $G_{k^{\prime}}^{i^{\prime}} \cap N=\emptyset$ whenever $k^{\prime} \neq k$.

Proof. In this case, $G^{i}=\bigcup_{k=1}^{n} G_{k}^{i}$ is also a compact family of the finite subsets of $\mathbb{N}$. To see this, choose a sequence $G^{i_{m}}, m \in \mathbb{N}$, out of $G^{i}: i \in I$ and find $M \in[\mathbb{N}]$ such that for any $k=1, \ldots, n,\left\{G_{k}^{i_{m}}: m \in M\right\}$ is convergent to some $G_{k}^{i}$. Then it is easy to check that $\left\{G^{i_{m}}\right\}_{m \in M}$ converges to $G^{i}=\bigcup_{k=1}^{n} G_{k}^{i}$.

Therefore, by Theorem 2.1, we can find $N=\left\{n_{1}<n_{2}<\ldots\right\} \in[L]$ such that given $i \in I, q \in \mathbb{N}, k \in\{1, \ldots, n\}$ and $A \subset G_{k}^{i} \cap\left\{n_{1}, \ldots, n_{q}\right\}$, there is $i_{q} \in I$ such that $G_{k}^{i_{q}} \cap\left\{n_{1}, \ldots, n_{q}\right\}=A$ and for all $k^{\prime} \neq k, G_{k^{\prime}}^{i_{q}} \cap\left\{n_{1}, \ldots, n_{q}\right\} \subset A$. Now choosing $M \in[\mathbb{N}]$ such that $\left\{\left(G_{k^{\prime}}^{i_{q}}\right)_{k=1, \ldots, n}\right\}_{q \in M}$ is convergent to some $\left(G_{k^{\prime}}^{j}\right)_{k^{\prime}=1, \ldots, n}$, it is easy to check that $G_{k}^{j} \cap N=A$ and for all $k^{\prime} \neq k, G_{k^{\prime}}^{j} \cap N \subset A$.

Remark 2.1. Using the notation of the above corollary, it is not clear to us even for $n=2$, whether there exists an $N \in[L]$ such that the following holds:

Given $i \in I$ and $A_{1} \subset G_{1}^{i} \cap N, A_{2} \subset G_{2}^{i} \cap N$, there is $i^{\prime} \in I$ such that $A_{1}=G_{1}^{i} \cap N$ and $A_{2}=G_{2}^{i} \cap N$. 


\section{Sequences With an unConditional Basic subsequence}

From Theorem 1.4 we obtain a proof of Theorem 1.3 as follows.

Proof of Theorem 1.3. Let $M>0$ be such that $\left(f_{m}\right)_{m}$ is uniformly bounded by $M$. By passing if necessary to a subsequence of $\left\{f_{m}: m \in \mathbb{N}\right\}$, we may assume that for all $m \in \mathbb{N}, \epsilon_{m}<\mu$. Consider the sequence $g_{m}: K \rightarrow \mathbb{R}^{n}$ with

$$
g_{m}(x)= \begin{cases}f_{m}(x), & \text { if }\left\|f_{m}(x)\right\| \geq \mu, \\ 0, & \text { otherwise }\end{cases}
$$

Since $\epsilon_{m}<\mu$, we have that $g_{m}$ is continuous for all $m \in \mathbb{N}$ and moreover $\| f_{m}-$ $g_{m} \| \leq \epsilon_{m} \rightarrow 0$. Therefore it suffices to find an unconditional basic subsequence for $\left\{g_{m}\right\}_{m \in \mathbb{N}}$.

For any $m \in \mathbb{N}$ and $1 \leq k \leq n$, let $g_{m}^{k}$ be the $k$-coordinate function of $g_{m}$. i.e. $g_{m}(x)=\left(g_{m}^{1}(x), \ldots, g_{m}^{n}(x)\right)$. Next for $x \in K$ and $1 \leq k \leq n$ we define the following subsets of $\mathbb{N}$ :

$$
G_{k, 1}^{x}=\left\{m \in \mathbb{N}: g_{m}^{k}(x) \geq \mu\right\}, \quad G_{k,-1}^{x}=\left\{m \in \mathbb{N}: g_{m}^{k}(x) \leq-\mu\right\} .
$$

Also let $G^{x}=\left\{m \in \mathbb{N}:\left\|g_{m}(x)\right\| \geq \mu\right\}$. Considering the supremum norm on $\mathbb{R}^{n}$, we have that $G^{x}=\bigcup_{i=-1,1} \bigcup_{k=1}^{n} G_{k, i}^{x}$.

It is easy to see that since $\left(g_{n}\right)_{n}$ are continuous and pointwise null, $\left\{G^{x}: x \in K\right\}$ is a compact family of finite subsets of $\mathbb{N}$.

By Theorem 1.4 there is $N=\left\{n_{1}<n_{2}<\ldots\right\} \in[\mathbb{N}]$ such that for all $q \in \mathbb{N}$, $x \in K, k=1, \ldots, n, i=-1,1$ and $A \subset G_{k, i}^{x} \cap\left\{n_{1}, \ldots, n_{q}\right\}$ there is $x^{\prime} \in K$ such that $G_{k, i}^{x^{\prime}} \cap\left\{n_{1}, \ldots, n_{q}\right\}=A$ and for all other combinations $\left(k^{\prime}, i^{\prime}\right) \neq(k, i), G_{k, i^{\prime}}^{x^{\prime}} \cap$ $\left\{n_{1}, \ldots, n_{q}\right\} \subset A$. Using this, we next prove that $g_{n_{1}}, g_{n_{2}}, \ldots$ is an unconditional basic sequence. So let $q \in \mathbb{N}, a_{n_{1}}, \ldots, a_{n_{q}} \in \mathbb{R}$ and $A \subset\left\{n_{1}, \ldots, n_{q}\right\}$. We try to estimate the norm of $\sum_{m \in A} a_{m} g_{m}$ in terms of the norm of $\sum_{\ell=1}^{q} a_{n_{\ell}} g_{n_{\ell}}$.

For some $x \in K$, we have that $\left\|\sum_{m \in A} a_{m} g_{m}\right\|=\left\|\sum_{m \in A} a_{m} g_{m}(x)\right\|$ and since $\left\|\sum_{m \in A} a_{m} g_{m}(x)\right\|=\sup \left\{\left|\sum_{m \in A} a_{m} g_{m}^{k}(x)\right|: k=1, \ldots, n\right\}$, for some $k=1, \ldots, n$ $\left\|\sum_{m \in A} a_{m} g_{m}\right\|=\left|\sum_{m \in A} a_{m} g_{m}^{k}(x)\right|$.

$$
\begin{gathered}
A_{1,1}=\left\{m \in A: a_{m} \geq 0, g_{m}^{k}(x)>0\right\}, \quad A_{1,-1}=\left\{m \in A: a_{m} \geq 0, g_{m}^{k}(x)<0,\right\}, \\
A_{-1,1}=\left\{m \in A: a_{m}<0, g_{m}^{k}(x)>0\right\}, \quad A_{-1,-1}=\left\{m \in A: a_{m}<0, g_{m}^{k}(x)<0\right\} .
\end{gathered}
$$

Since $A=\bigcup_{i=-1,1} \bigcup_{j=-1,1} A_{j, i}$, we get that for appropriate choose of $i$ and $j$,

$$
\left\|\sum_{m \in A} a_{m} g_{m}\right\|=\left|\sum_{m \in A} a_{m} g_{m}^{k}(x)\right| \leq 4\left|\sum_{m \in A_{j, i}} a_{m} g_{m}^{k}(x)\right| .
$$

Therefore

$$
\left\|\sum_{m \in A} a_{m} g_{m}\right\| \leq 4\left|\sum_{m \in A_{j, i}} a_{m} g_{m}^{k}(x)\right| .
$$

Obviously, $A_{j, i} \subset G_{k, i}^{x} \cap\left\{n_{1}, \ldots, n_{q}\right\}$. Therefore we can find $x^{\prime} \in K$ such that $G_{k, i}^{x^{\prime}} \cap\left\{n_{1}, \ldots, n_{q}\right\}=A_{j, i}$ and for all other $\left(k^{\prime}, i^{\prime}\right) \neq(k, i), G_{k^{\prime}, i^{\prime}}^{x^{\prime}} \cap\left\{n_{1}, \ldots, n_{q}\right\} \subset$ $A_{j, i}$. Note that in particular $G_{k,-i}^{x^{\prime}} \cap\left\{n_{1}, \ldots, n_{q}\right\}=\emptyset$, since a value of a function 
cannot be both positive and negative. Therefore setting $T_{q}=\left\{n_{1}, \ldots, n_{q}\right\}$, we have

$$
\begin{aligned}
\left\|\sum_{\ell=1}^{q} a_{n_{\ell}} g_{n_{\ell}}\right\| & \geq\left|\sum_{\ell=1}^{q} a_{n_{\ell}} g_{n_{\ell}}^{k}\left(x^{\prime}\right)\right|=\left|\sum_{m \in G_{k, i}^{x^{\prime}} \cap T_{q}} a_{m} g_{m}^{k}\left(x^{\prime}\right)+\sum_{m \in G_{k,-i}^{x^{\prime}} \cap T_{q}} a_{m} g_{m}^{k}\left(x^{\prime}\right)\right| \\
& =\left|\sum_{m \in A_{j, i}} a_{m} g_{m}^{k}\left(x^{\prime}\right)\right|=\sum_{m \in A_{j, i}}\left|a_{m}\right|\left|g_{m}^{k}\left(x^{\prime}\right)\right| \geq \sum_{m \in A_{j, i}}\left|a_{m}\right| \mu \\
& =\frac{\mu}{M} \sum_{m \in A_{j, i}}\left|a_{m}\right| M \geq \frac{\mu}{4 M} 4\left|\sum_{m \in A_{j, i}} a_{m} g_{m}^{k}(x)\right| \\
& \geq \frac{\mu}{4 M}\left\|\sum_{m \in A} a_{m} g_{m}\right\|
\end{aligned}
$$

which completes the proof.

Proof of Theorem 1.2. To avoid confusion, we denote by $\|\cdot\|_{X}$ the norm of $X$, and by $\|\cdot\|$ the norm on $C(K, X)$, where for an $f \in C(K, X),\|f\|=\sup \left\{\|f(k)\|_{X}: k \in K\right\}$.

Let $f_{n}(K)=\left\{w_{n}^{1}, w_{n}^{2}, \ldots, w_{n}^{J_{n}}\right\}$ with $J_{n} \leq J$ be an enumeration of $f_{n}(K)$ such that $w_{n}^{1}, w_{n}^{2}, \ldots, w_{n}^{J_{n}}$ are different from each other and moreover $\left\|w_{n}^{1}\right\|_{X} \leq\left\|w_{n}^{2}\right\|_{X} \leq$ $\cdots \leq\left\|w_{n}^{J_{n}}\right\|_{X}$. By the Pigeonhole Principle, we get that for some $m \leq J$ and for infinitely many $n, J_{n}=m$. Since each $f_{n}$ is normalized, for all those $n,\left\|w_{n}^{J_{n}}\right\|_{X}=1$. Therefore there exists an $m$ such that $\left\|w_{n}^{m-1}\right\|_{X}$ forms a null sequence, but $\left\|w_{n}^{m}\right\|_{X}$ does not. Using this observation we next argue that we may assume, passing if necessary to a different sequence, that there is a $\mu>0$ such that for all $n, w_{n}^{1}=0$ and $\left\|w_{n}^{2}\right\|_{X} \geq \mu$.

Set $\epsilon_{n}=\left\|w_{n}^{m-1}\right\|_{X} \rightarrow 0$. By passing if necessary to a subsequence of $\left(f_{n}\right)_{n}$ we may assume that there is a $\mu>0$ such that for all $n, \epsilon_{n}<\mu \leq\left\|w_{n}^{m}\right\|_{X}$. Now define $g_{n}: K \rightarrow X$ as follows:

$$
g_{n}(k)= \begin{cases}0, & \text { if }\left\|f_{n}(k)\right\|_{X} \leq \epsilon_{n} \\ f_{n}(k), & \text { otherwise }\end{cases}
$$

It is easy to see that in this case every $g_{n}$ is continuous, the cardinality of $g_{n}(K)$ is also bounded by $J$ and for every $k \in K$ either $g_{n}(k)=0$ or $\left\|g_{n}(k)\right\|_{X} \geq \mu$. Moreover, all but finitely many $g_{n}$ take the value $0, \operatorname{since}\left(f_{n}\right)_{n}$ is pointwise null. Observe that $\left\|f_{n}-g_{n}\right\| \leq \epsilon_{n} \rightarrow 0$. Therefore the existence of an unconditional subsequence of $\left(g_{n}\right)_{n}$ implies the existence of an unconditional subsequence of $\left(f_{n}\right)_{n}$. So we may indeed assume that for all $n, w_{n}^{1}=0$ and $\left\|w_{n}^{2}\right\|_{X} \geq \mu$.

For any $r \in[2, J]$ and $k \in K$, let $G_{r}^{k}=\left\{n \in \mathbb{N}: r \leq J_{n}\right.$ and $\left.f_{n}(k)=w_{n}^{r}\right\}$. Also set $G^{k}=\bigcup_{r=2}^{J} G_{r}^{k}=\left\{n \in \mathbb{N}:\left\|f_{n}(k)\right\|_{X} \geq \mu\right\}$. Since $\left(f_{n}\right)_{n \in \mathbb{N}}$ is a pointwise null sequence of continuous functions and for every $k \in K$, either $f_{n}(k)=0$ or $\left\|f_{n}(k)\right\|_{X} \geq \mu$, we get that $\left\{G^{k}: k \in K\right\}$ is a compact family of finite subsets of $\mathbb{N}$. We use Theorem 1.4 to obtain an $N=\left\{n_{1}<n_{2}<\ldots\right\} \in[\mathbb{N}]$ such that for any $k \in K, 2 \leq r \leq J, q \in \mathbb{N}$ and $A \subset G_{r}^{k} \cap\left\{n_{1}, \ldots, n_{q}\right\}$, there is $k^{\prime} \in K$ such that $G_{r}^{k^{\prime}} \cap\left\{n_{1}, \ldots, n_{q}\right\}=A$ and for all $r^{\prime} \neq r, G_{r^{\prime}}^{k^{\prime}} \cap\left\{n_{1}, \ldots, n_{q}\right\} \subset A$. Observe that since for $r^{\prime} \neq r, w_{n}^{r^{\prime}} \neq w_{n}^{r}$, we have that $G_{r}^{k^{\prime}} \cap G_{r^{\prime}}^{k^{\prime}}=\emptyset$ and therefore in this case $G_{r^{\prime}}^{k^{\prime}} \cap\left\{n_{1}, \ldots, n_{q}\right\}=\emptyset$. 
We next prove that $\left(f_{n}\right)_{n \in N}$ is an unconditional basic sequence. So let $q \in$ $\mathbb{N}, a_{n_{1}}, a_{n_{2}}, \ldots, a_{n_{q}} \in \mathbb{R}$ and $A \subset\left\{n_{1}, \ldots, n_{q}\right\}$. We try to estimate the norm of $\sum_{n \in A} a_{n} f_{n}$ in terms of the norm of $\sum_{\ell=1}^{q} a_{n_{\ell}} f_{n_{\ell}}$.

For some $k \in K$,

$$
\left\|\sum_{n \in A} a_{n} f_{n}\right\|=\left\|\sum_{n \in A} a_{n} f_{n}(k)\right\|_{X} .
$$

Let $A=\bigcup_{r=2}^{J} A_{r}$ where $A_{r}=\left\{n \in A: r \leq J_{n}\right.$ and $\left.f_{n}(k)=w_{n}^{r}\right\}$. Since for $r \neq r^{\prime}$, $w_{n}^{r} \neq w_{n}^{r^{\prime}}$ we get that $A_{r}, r=2, \ldots, J$, are pairwise disjoint. Therefore there is an $r_{0} \in[2, J]$ such that

$$
\left\|\sum_{n \in A} a_{n} f_{n}(k)\right\|_{X} \leq(J-1)\left\|\sum_{n \in A_{r_{0}}} a_{n} f_{n}(k)\right\|_{X}=(J-1)\left\|\sum_{n \in A_{r_{0}}} a_{n} w_{n}^{r_{0}}\right\|_{X} .
$$

Since obviously $A_{r_{0}} \subset G_{r_{0}}^{k} \cap\left\{n_{1}, \ldots, n_{q}\right\}$, by our hypothesis for $N$ there exists $k^{\prime} \in K$ such that $G_{r_{0}}^{k^{\prime}} \cap\left\{n_{1}, \ldots, n_{q}\right\}=A_{r_{0}}$ and for $r^{\prime} \neq r_{0}, G_{r^{\prime}}^{k^{\prime}} \cap\left\{n_{1}, \ldots, n_{q}\right\}=\emptyset$. In this case for all $n \in\left\{n_{1}, \ldots, n_{q}\right\}$ if $f_{n}\left(k^{\prime}\right) \neq 0$, then $f_{n}\left(k^{\prime}\right)=w_{n}^{r_{0}}$. Therefore

$$
\left\|\sum_{\ell=1}^{q} a_{n_{\ell}} f_{n_{\ell}}\left(k^{\prime}\right)\right\|_{X}=\left\|\sum_{n \in A_{r_{0}}} a_{n} w_{n}^{r_{0}}\right\|_{X},
$$

so that by (6) and (5) we get

$$
\left\|\sum_{n \in A} a_{n} f_{n}\right\| \leq(J-1)\left\|\sum_{\ell=1}^{q} a_{n_{\ell}} f_{n_{\ell}}\right\|
$$

and this proves immediately that $\left(f_{n}\right)_{n \in N}$ is an unconditional basic sequence.

Remark 3.1. Maurey-Rosenthal's example [8] shows that there is a compact $K$ such that for a pointwise null sequence $f_{n} \in C(K),\left\{f_{n}\right\}_{n}$ has no unconditional basic subsequence. In this case the range of every $f_{n}$ is a countable set $\{0\} \cup\left\{r_{k}: k \in \mathbb{N}\right\}$ where $\left\{r_{k}\right\}_{k}$ is a null sequence. As we have mentioned in the Introduction, a slight modification of $\left(f_{n}\right)_{n}$ derives a sequence $\left(g_{n}\right)_{n}$ pointwise convergent to zero with no unconditional subsequence and such that every $g_{n}$ has a finite range.

\section{ACKNOWLEDGMENTS}

I thank Professors S.A. Argyros and S. Mercourakis for their useful suggestions on this paper.

\section{REFERENCES}

[1] S. A. Argyros, G. Godefroy, and H. P. Rosenthal. Descriptive set theory and Banach spaces. In Handbook of the geometry of Banach spaces, Vol. 2, pages 1007-1069. North-Holland, Amsterdam, 2003. MR.1999190 (2004g:46002)

[2] S. A. Argyros, S. Mercourakis, and A. Tsarpalias. Convex unconditionality and summability of weakly null sequences. Israel J. Math., 107:157-193, 1998. MR.1658551 (99m:46021)

[3] E. Ellentuck. A new proof that analytic sets are Ramsey. J. Symbolic Logic, 39:163-165, 1974. MR0349393 (50:1887)

[4] J. Elton. Weakly null normalized sequences in Banach spaces. Ph.D. thesis, 1978.

[5] F. Galvin and K. Prikry. Borel sets and Ramsey's theorem. J. Symbolic Logic, 38:193-198, 1973. MR0337630 (49:2399)

[6] I. Gasparis, E Odell, and B. Wahl. Weakly null sequences in the Banach space $C(K)$. Preprint.

[7] J. Lopez-Abad and S. Todorcevic. Unconditional subsequences of weakly null sequences. In preparation, 2004. 
[8] B. Maurey and H. P. Rosenthal. Normalized weakly null sequence with no unconditional subsequence. Studia Math., 61(1):77-98, 1977. MR0438091(55:11010)

[9] E. Odell. Applications of Ramsey theorems to Banach space theory. In Notes in Banach spaces, pages 379-404. Univ. Texas Press, Austin, Tex., 1980. MR0606226 (83g:46018)

[10] J. Silver. Every analytic set is Ramsey. J. Symbolic Logic, 35:60-64, 1970. MR0332480 $(48: 10807)$

[11] S. Todorcevic. High-dimensional Ramsey theory. Preprint, CRM, 2004.

Department of Mathematics, National Technical University of Athens, Zografou Campus, 15780, Athens, Greece

E-mail address: aarva@math.ntua.gr 